\title{
Suitability of chlorhexidine impregnated dressings on dialysis catheters in an acute dialysis setting: Lessons from our experience
}

\author{
Edward Zimbudzi
}

Department of Nephrology, Southern Health, Monash Medical Centre, Clayton, Melbourne, Victoria, Australia

\begin{abstract}
Haemodialysis Catheter-Related Infections (HCRIs) are a major cause of mortality and morbidity among the dialysis population and they have also overburdened the health care systems financially. Due to this problem, many dialysis units are keen to implement any evidence-based procedure that could reduce the occurrence of HCRIs. One such procedure is the dressing of haemodialysis permacath exit sites. The purpose of this study was to find out if there were any significant benefits of using chlorhexidine impregnated patches (biopatch ${ }^{\mathrm{TM}}$ ) to dress permacath exit sites in an acute dialysis setting of a metropolitan teaching hospital.
\end{abstract}

Utilizing an observational longitudinal study design, fourteen participants with permacaths were conveniently sampled. Permacath exit sites were dressed with biopatch dressings which were changed after every 7 days and more often if the exit site showed signs of redness or bleeding. Post biopatch trial, all the patients who participated in this trial were followed up for three months. During the follow up period, permacath exit sites were cleaned with chlorhexidine $2 \%$ and then mupirocin (bactroban) ointment was applied as per unit's protocol followed by an application of an IV 3000 dressing to cover the exit site. The dressing was changed three times a week.

Bleeding of catheter site resulted in removal of $19 \%$ of the dressings before the standard 7 day period and $37 \%$ of the patches were changed prematurely due to suspected exit site infection. Only $2 \%$ of the patches were taken off due to exit site sensitivity to the biopatch ${ }^{\mathrm{TM}}$. There were 15 patches $(35 \%)$ which were removed after

\section{Corresponding Author}

Mr Edward Zimbudzi

Department of Nephrology, Monash Medical Centre, Clayton, VIC 3168, Australia

Email: edward.zimbudzi@southernhealth.org.au 
7 days and $7 \%$ of the patches were not followed up. Overall, dressings were left intact for a mean period of 4.8 days $\mathrm{SD} \pm 1.8$. One case of suspected Catheter Associated Blood Stream Infection (CABSI) was reported during the three months post biopatch use.

The results of this trial demonstrate that using biopatch ${ }^{\mathrm{TM}}$ in an acute dialysis unit may not produce anticipated positive results to the patient and the economic benefit to the health service may be minimal.

\section{Key words}

Chlorhexidine, therapeutic use; occlusive dressing; renal dialysis; catheter-related infections, therapy; equipment contamination

\section{Introduction}

A biopatch ${ }^{T M}$ is a dressing made up of polyurethane absorptive foam with chlorhexidine gluconate, ${ }^{1}$ a well known antiseptic agent with antimicrobial and antifungal properties. Biopatches are used on wounds with percutaneous medical devices such as catheters. Dressing permacath with biopatch ${ }^{\mathrm{TM}}$ is meant to reduce incidences of exit site infection $(\mathrm{ESI}) .{ }^{2}$ Besides that, the patches are designed in such a way that they can absorb excess exudate. The fact that the dressings are changed after a week makes biopatch ${ }^{\mathrm{TM}}$ dressings cost effective. The Monash Medical Center in-center dialysis unit carried out a biopatch ${ }^{\text {TM }}$ trial from January to March 2011. The objective of this trial was to find out if there were any significant benefits of using biopatch to dress permacath exit sites in an acute dialysis setting.

\section{Method}

The study was undertaken in the acute renal unit of Monash Medical Center (MMC), a large metropolitan teaching hospital in the south east of Melbourne. An observational longitudinal study design was used for this study. Participants were conveniently sampled. Patients who had a permacath that was currently in use met the inclusion criteria for this study.

Prior to commencement of the biopatch ${ }^{T M}$ trial, dialysis staff were educated on how to apply the dressing. Permacath sites were cleaned with chlorhexidine $2 \%$ as per hospital protocol before chlorhexidine impregnated patches were left in situ. Sandwich dressings comprising two IV 3000 were done to secure the patches. Patches were meant to stay intact for a period of 7 days according to the manufacturer's recommendations, but in this study they were removed prematurely if there was bleeding from the catheter exit site or if there was at least one sign of exit site infection such as redness, tenderness, drainage, chills and fever. Exit site infections were managed as per hospital policy which required patients to have a septic screen to confirm source of infection. Patches were also removed prematurely if there was suspected skin reaction evidenced by severe burning, itching, and redness, blistering, peeling, swelling and rash. Application of the patches was avoided on patients who had just had the insertion of the permacaths due to increased risk of bleeding.

Fourteen patients participated in this trial and 43 patches were used among these patients. All the patients who participated in this trial were followed up for three months. By then, they were using the unit's conventional dressing. Permacath exit sites were cleaned with chlorhexidine $2 \%$ and then mupirocin (bactroban) ointment was applied as per unit's protocol. This was followed by an application of an IV 3000 dressing to cover the exit site. The dressing was changed three times a week.

\section{Results}

Figure 1 describes the results of the 43 chlorhexidine impregnated patches which were used during the three months trial. Bleeding of catheter site resulted in the removal of $19 \%$ of the dressings before the standard 7 day period. Suspected infection of the exit site resulted in premature removal of $37 \%$ of the patches. Only $2 \%$ of the patches were taken off due to exit site sensitivity to the biopatch. There were 15 patches (35\%) which were removed after 7 days. It was difficult to follow up 
$7 \%$ of the patches since they were removed at other dialysis units where the patients were transferred.

During the use of biopatch, no Catheter Associated Blood Stream Infections (CABSIs) were reported. However, one case of suspected CABSI was reported during the three months post biopatch use and this was consistent with an overall HCRI incidence of $4 \%$ in the dialysis unit for the year 2011. In terms of costs, the biopatch dressing was six times more expensive than the unit's conventional dressing during this trial period.

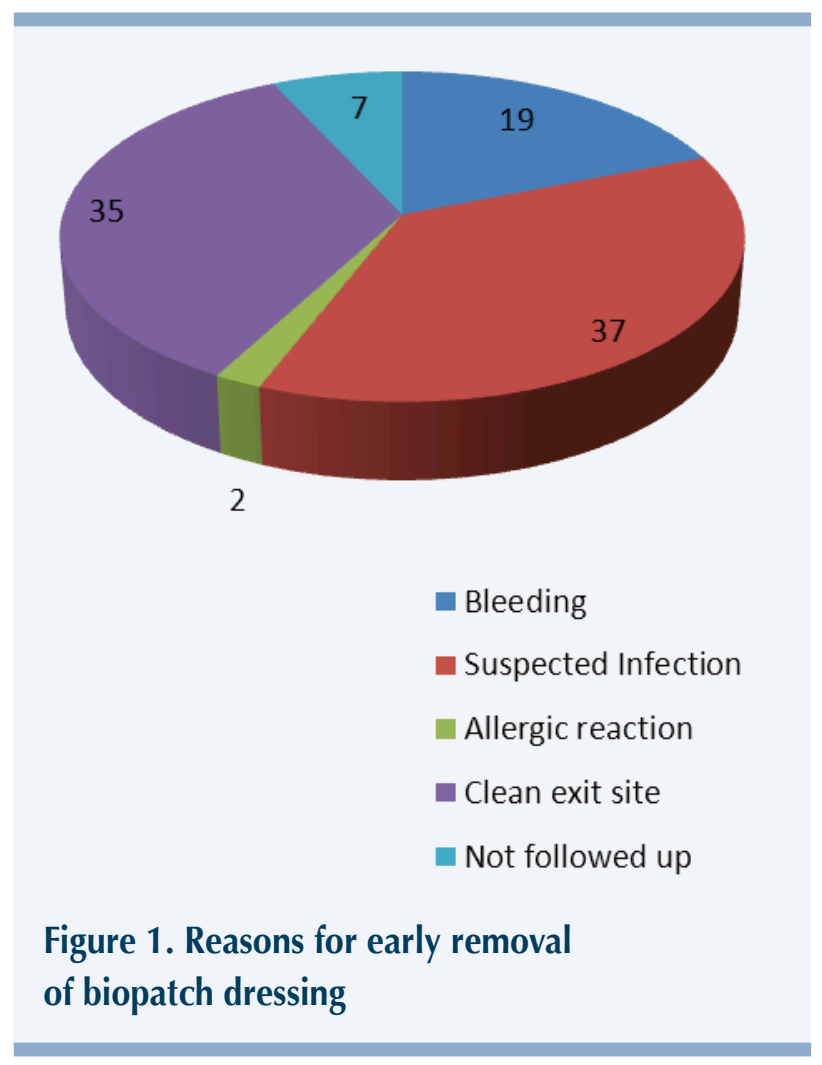

\section{Discussion}

One of the benefits of using biopatch ${ }^{\text {TM }}$ is that the dressing requires to be changed less frequently (after 7 days) without posing infection risks to the patient. ${ }^{3}$ Given that biopatch ${ }^{\mathrm{TM}}$ dressings cost considerably more than most dressings used for permacath exit site, the reduced frequency of dressing change renders them cost effective. However, in our trial only $35 \%$ of the patches were changed after 7 days. According to Timsit et al. extending the theoretical dressing change interval from 3 to 7 days resulted in only $9 \%$ decrease in the number of changes per catheter-day. ${ }^{3}$ The benefit of changing dressings less frequently with the use of biopatch cannot therefore be fully exploited if the majority of dressings require to be changed before their standard time.

About $37 \%$ of the biopatches in our study were removed because of suspected exit site infection. Exit site infection was deemed to have occurred if a patient presented with any one of the signs of infection such as redness, tenderness, drainage, chills and fever. As demonstrated by other studies, ${ }^{4}$ exit site appearance cannot be relied on to identify catheter related colonization or Central Venous Catheter (CVC)-related Blood Stream Infections (BSI). In this study, exit site redness could have been caused by sensitivity to biopatch $^{\mathrm{TM}}$ and trauma among other causes.

While chlorhexidine impregnated sponges can absorb exudate from the exit site, our experience shows that 1 in 5 dressings were changed due to bleeding from the exit site. Although dressing with biopatch ${ }^{\mathrm{TM}}$ was avoided immediately post catheter insertion, some haemodialysis catheters continued to bleed even a few days after catheter insertion. In these instances, other catheter site dressing regimens such as gauze dressing were preferred. ${ }^{5}$

Biopatches undoubtedly play a pivotal role in reducing ESIs and BSIs but their routine use with dialysis catheters requires additional studies. The use of a chlorhexidine-impregnated foam dressing (biopatch $^{\mathrm{TM}}$ ) did not decrease catheter-related BSIs among hemodialysis patients with tunnelled central venous catheters. ${ }^{6}$ Other studies have demonstrated benefits of biopatch ${ }^{T M}$ in patients with short-term, but high risk intravascular catheters ${ }^{7,8}$ while the benefits of biopatch ${ }^{\mathrm{TM}}$ in long term catheters used for dialysis have not been well documented.

On another note, our unit's conventional dressing practice involved the routine use of mupirocin which is not recommended by some current guidelines. However, some recent randomized controlled trials (RCTs) have produced data which shows that routine application of mupirocin reduces the risk of catheter related bacteremia and prolongs catheter life. ${ }^{9}$ Information available from the Caring for Australians with Renal Impairment guidelines (CARI) on this subject is out of date, but these guidelines recommended the application of mupirocin ointment on catheter exit site 
to reduce local and systemic infection ${ }^{10}$ and probably that explains why over $20 \%$ of Australian dialysis units are still using mupirocin ointment. ${ }^{11}$

This study has several limitations. The sample sizes for the number of patients recruited and biopatches used is small to produce statistically significant results. Furthermore, the study relied on subjective data especially on determination of whether the catheter exit sites were infected or not. In this regard, the author recommends a more objective study which utilizes a bigger sample size to give adequate power to obtain statistically significant results.

\section{Conclusion}

The results of this trial demonstrate that using biopatch $^{\mathrm{TM}}$ in an acute dialysis unit may not produce anticipated positive results to the patient and the economic benefit to the health service may be minimal. Comparing the present practice of changing the dressings three times a week and applying mupirocin to the use of biopatch ${ }^{\mathrm{TM}}$, the current practice seems to be cost effective. Apart from that, our current dressing regimen gives full visibility of the insertion site so infections can easily be identified.

\section{Acknowledgements}

The author would like to thank the marketing department of Johnson \& Johnson, Australia for providing MMC in-center dialysis unit with biopatches for trial. This study would not have been a success without the sterling work put forward by the MMC incenter dialysis nursing staff during the trial period.

\section{References}

1. Maki DG, Mermel L, Genthner D, Hua S, Chiacchierini RP. An evaluation of BIOPATCH. Antimicrobial Dressing compared to routine standard of care in the prevention of catheterrelated blood stream infection. Johnson and Johnson Medical, Division of ETHICON, Inc. 2000.

2. Onder AM, Chandler J, Coakley S, Francoeur D, Abitbol C, Zillerueelo G. Controlling exit site infections: does it decrease the incidence of catheter related bacteremia in children on chronic hemodialysis? Hemodia Int 2009; 13(1): 11-18. http:// dx.doi.org/10.1111/j.1542-4758.2009.00348.x

3. Timsit J-F, Schewable C, Bouadama L, et al. Chlorhexidine impregnated sponges and less frequent dressing changes for prevention of catheter related infections in critically ill adults: A randomized controlled trial. JAMA 2009; 301(12): 12311241. http://dx.doi.org/10.1001/jama.2009.376

4. Safdar N, Maki DG. Inflammation at the insertion site not predictive of CRBSI with short-term, non cuffed CVCs. Critical Care Medicine 2002; 30(12): 2632-2635. http://dx.doi. org/10.1097/00003246-200212000-00003

5. O'Grady NP, Alexander M, Dellinger PE, et al. Guidelines for the prevention of intravascular catheter- related infections. Infection Control and Hospital Epidemiology 2002; 23(12): 759-769. http://dx.doi.org/10.1086/502007

6. Camins BC, Richmond AM, Dyer KL, et al. A crossover intervention trial evaluating the efficacy of a chlorhexidineimpregnated sponge in reducing catheter-related bloodstream infections among patients undergoing hemodialysis. Infect Control Hosp Epidemiol 2010; 31(11): 1118-1123. http:// dx.doi.org/10.1086/657075

7. Ruschulte $\mathrm{H}$, Franke $\mathrm{M}$, Gastmeier $\mathrm{P}$, et al. Prevention of central venous catheter related infections with chlorhexidine gluconate impregnated wound dressings: a randomized controlled trial. Ann Hematol 2009; 88: 267-272. http:// dx.doi.org/10.1007/s00277-008-0568-7

8. Mann TJ, Orlikowski CE, Gurrin LC, Keil AD. The effect of the biopatch, a chlorhexidine impregnated dressing on bacterial colonisation of epidural catheter exit sites. Anaesth Intensive Care 2001; 29(6): 600-603.

9. McCann M, Moore ZE. Interventions for preventing infectious complications in haemodialysis patients with central venous catheters. Cochrane Database Systematic Review 2010; 20(1): CD00689.

10. Caring for Australian with Renal Impairment (CARI) guidelines. CARI Vascular Access Guidelines. CARI. 2000. Retrieved $3^{\text {rd }}$ of June, 2012 from http://www.cari.org.au/DIALYSIS_va_ published/Preventing_Staphylococcal_related.pdf.

11. Bennett PN, Janko A, Whittington T. Central Venous Dialysis Catheter Care: An Australian Survey. Ren Soc Aust I 2005; 1(2): 50-57. 